\title{
Predicting risk and outcomes for frail older adults: a protocol for an umbrella review of available frailty screening tools
}

\author{
João Apóstolo ${ }^{1}$ \\ Richard Cooke ${ }^{2}$ \\ Elzbieta Bobrowicz-Campos ${ }^{1}$ \\ Silvina Santana ${ }^{3}$ \\ Maura Marcucci ${ }^{4}$ \\ Antonio $\mathrm{Cano}^{5}$ \\ Miriam Vollenbroek ${ }^{6}$ \\ Carol Holland ${ }^{2}$
}

1 Health Sciences Research Unit: Nursing, Nursing School of Coimbra, Portugal Center for Evidence-Based Practice: a Collaborating Center of the Joanna Briggs Institute

2 Aston Research Centre for Healthy Ageing (ARCHA), Aston University, Birmingham, United Kingdom

3 Economics, Management and Industrial Engineering Department, University of Aveiro, Portugal

4 Geriatric Unit, Fondazione IRCCS Ca' Granda Ospedale Maggiore Policlinico and Department of Clinical Science and Community Health, University of Milan, Milan, Italy

5 Department Of Paediatrics, Obstetrics and Gynaecology, Universitat de Valência, Spain

6 Roessingh Research and Development, The Netherlands

\section{Corresponding author: \\ João Apóstolo \\ apostolo@esenfc.pt}

\section{Review question/objective}

The aim of this systematic review is to comprehensively search the available literature and to summarize the best available evidence from systematic reviews in relation to published screening tools to identify pre-frailty and frailty in older adults, that is: (i) to determine their psychometric proprieties; (ii) to assess their capacity to detect pre-frail and frail conditions against established methods; and (iii) to evaluate their predictive ability.

More specifically, the review will focus on the following questions:

- What is the reliability and validity of existing screening tools that assess pre-frailty/frailty in older patients? 
- How sensitive and specific are pre-frailty/frailty assessment tools in identifying patients with poor prognosis?

- What is the ability of available pre-frailty/frailty assessment tools to predict adverse health outcomes such as functional disability, hospitalization, institutionalization, comorbidities and death?

\section{Background}

Frailty is an age-related state of vulnerability resulting from a balance between the maintenance of health and the deficits threatening it. ${ }^{1,2}$ This clinical condition compromises the ability to cope with daily or acute stressors and, further, increases the risk of adverse outcomes, predisposing those involved to disability and dependency on others for daily life activities, and leading to hospitalization and institutional placement. ${ }^{3,4}$ It is also a predictor of higher mortality rates. ${ }^{4-7}$ In the absence of biological markers, an operational definition of frailty has been proposed. ${ }^{2,8}$ This definition is based on physical markers, including weakness with low muscle strength (e.g. poor grip strength), overall slowness (particularly of gait), decreased balance and mobility, fatigability or exhaustion, low physical activity and involuntary weight loss. For diagnostic purposes, at least three of these symptoms must be observed. ${ }^{8}$ The presence of only one or two of them indicates the earlier stage of frailty, namely, pre-frailty. Despite high predictive validity of this operational definition, and despite its common use in clinical settings, many researchers believe it is insufficient, asserting that it should also include cognitive and mental health domains, and possibly also social domains such as living alone. ${ }^{9-12}$ Other dimensions recognized as important for identifying frailty are the ability to deal with activities of daily living and quality of life, as for individuals with this clinical condition both of these areas tend to be decreased. $^{9,13}$

This lack of consensus on the definition of frailty (based on physical markers as opposed to a broader multidimensional approach) is also reflected in divergences related to the prevalence data obtained from epidemiological studies. Systematic comparison of these data ${ }^{14}$ shows that frailty prevalence differs from $4 \%$ to $17 \%$ in the population aged 65 and over, and in case of pre-frailty, prevalence varies from $19 \%$ to $53 \%$ of the same age group, with average values of $10.7 \%$ and $41.6 \%$, respectively. The divergences between estimates are also conditioned by demographic variables such as age and gender. Namely, for elders aged 80-84 the prevalence of frailty is estimated as $15.7 \%$, and for elders over the age of $84,26.1 \%$. Additionally, women tend to have higher rates of frailty than men.

Although the condition of frailty has been studied for years, there is no consensus on its pathophysiologic mechanism. According to some authors ${ }^{2,8,15}$, this state of increased vulnerability is due to accumulation of subthreshold decrements in physiologic reserves that affects multiple physiologic systems. Other authors ${ }^{16,17}$ have described frailty in terms of progressive dysregulation in a number of main physiologic systems and their complex interconnected network, and subsequent depletion of homeostatic reserve and resiliency. Recently, discussion on the psychopathological mechanism of this clinical condition has been enriched by new theoretical proposals associating frailty to reduced capacity to compensate ageing-related molecular and cellular damage. ${ }^{13,18}$ In all these approaches it is assumed that the development of frailty may be modulated by disease. In other words, it can be precipitated or exacerbated by the occurrence of comorbid pathological conditions. ${ }^{19-}$ ${ }^{21}$ It is also suggested that increased vulnerability for adverse health outcomes can precede the onset of chronic diseases. ${ }^{19,20}$ However, according to Bergman et $\mathrm{al}^{19}$, it is probable that in this case, frailty is just a manifestation of subclinical and undiagnosed stages of such diseases. 
Because of the high prevalence and the severity of adverse outcomes of frailty, its screening should be a priority in appropriate components of primary care networks (including general practice, geriatrics, psychology, etc.), as well as in institutional or community care settings. Early diagnosis of this clinical condition can help improve care for older adults, making possible the minimization of the risk of pre-frail states developing into frail states (primary prevention), and implementation of therapeutic measures in order to attenuate or delay underlying conditions and symptoms, or to ameliorate the impact on independence or healthy and engaged lifestyles (loss of which would in turn have a further impact on frailty development, i.e. secondary prevention). ${ }^{2,4}$ In more advanced stages, frailty assessment provides valuable data necessary to plan and implement intervention strategies oriented to the preservation of functional status or to control the progression of adverse outcomes, such as recurrent hospitalizations, institutionalization or death (tertiary prevention). ${ }^{2,4}$ The evidence obtained from the implementation of various types of interventions for frailty indicates that the frailty condition can be managed and reduced. ${ }^{22-25}$ Screening for frailty can also provide information on populations at high risk of disability and poor prognosis, and help to identify reversible risk factors. ${ }^{2}$ These data are especially important for determining variables that make specific interventions more beneficial to specific patients.

In order to identify individuals at risk of frailty, several assessment tools have been developed. The most widely cited focus on physical markers of frailty ${ }^{2,8}$ or are based on the accumulation of deficits from physical, cognitive, mental health and functional domains. ${ }^{13,26}$ However, both types of measures seem to be insufficient. The first one does not cover all dimensions of frailty and consequently does not provide indications useful to treatment choice and care planning, and the last one is time consuming and thus is difficult to integrate into day-to-day health care practice. ${ }^{27}$ In more recent approaches, the indices created for frailty assessment integrate demographic, medical, social and functional information, and demonstrate their usefulness either for diagnostic purposes or to predict adverse health outcomes. ${ }^{28}$ According to the literature, there are more than 20 different measures being used for frailty screening. Nonetheless, it is still unknown how their characteristics match different samples within the frail/pre-frail condition and robust populations, and what is the best fit between these measures, purposes (e.g. to predict need for care, mortality or potential response to intervention) and contexts/populations to assess frailty in older age. Also, the reliability and validity of these measures need to be clarified, as well as the comparative sensitivity and specificity in identifying patients at risk of a poor prognosis.

A scoping search identified a large number of relevant systematic reviews; however in most cases they are confined to specific assessment measures related to a specific clinical model (phenotype model ${ }^{8}$, cumulative deficits model ${ }^{13}$ and predictive model ${ }^{28}$ ). For a clear view and objective evaluation of existing tools, this set of evidence needs to be systematized, compared and synthesized. In other words, it is essential to conduct an umbrella review.

A preliminary search of the JBI Database of Systematic Reviews and Implementation Reports, the Cochrane Database of Systematic Reviews, Prospero, CINAHL and Medline has revealed that there is currently no overview of reviews or umbrella review (neither published nor in progress) on this topic of sufficient reliability, validity and capacity to detect pre-frail and frail conditions, and with predictive accuracy of available screening tools for frailty in older adults ${ }^{29}$

The main goal of this umbrella review is to consolidate the available evidence regarding screening for pre-frailty and frailty from the published literature. More specifically, reviews will be summarized in order to determine the quality of screening tools in terms of frailty diagnosis and frailty prognosis. 


\section{Keywords}

Frail; elderly; pre-frailty; frailty; screening; diagnostic test accuracy; reliability and validity; predictive ability; umbrella review protocol

\section{Inclusion criteria}

\section{Types of participants}

This umbrella review will consider systematic reviews that include older adults (male and female), aged 65 years or over, in primary care networks (general practice, geriatrics, psychology, etc.) and in institutionalized or community care settings.

Because of the aims of this review (to determine the utility of screening in terms of detecting or predicting risk of currently available tools that assess pre-frailty and frailty in older adults in terms of outcome prognostics), various definitions of frailty will be considered: (i) focused on physical markers of frailty ${ }^{2,8}$, (ii) based on the accumulation of deficits from physical, cognitive, mental health and functional domains ${ }^{13,26}$, and (iii) integrating demographic, medical, psychological, social and functional information. $^{28}$

\section{Interventions}

The focus of this umbrella review will be systematic reviews that have evaluated the reliability and validity of available screening tools for pre-frailty and frailty in older adults, and that have assessed the utility of these tools to detect or predict risk for the onset/development of frailty or for other adverse events.

The capacity to detect pre-frail and frail conditions and predictive accuracy of the index tests will be compared against reference tests from the Cardiovascular Health Study (CHS) phenotype model ${ }^{8}$ and the Canadian Study of Health and Aging (CSHA) cumulative deficit model ${ }^{13}$ or other recognized gold standards (example, Comprehensive Geriatric Assessment $[\mathrm{CGA}]^{30}$ ).

\section{Types of outcomes}

This umbrella review will consider reviews that include the following outcome measures:

(i) Reliability of frailty screening tools defined in terms of internal consistency and repeatability (testretest) of findings.

(ii) Criterion validity of frailty screening tools defined as a measure of how well one variable or set of variables predicts an outcome based on information from other variables, as well as construct validity defined as the degree to which a test measures what it claims or purports to measure.

(iii) Sensitivity and specificity determined by comparison with the reference test (CHS, CSHA or CGA). Also, positive predictive values (PPV), negative predictive values (NPV) and likelihood ratios (LRs) will be considered.

(iv) Predictive accuracy of frailty screening tools for risks of adverse health outcomes, including functional disability, hospitalization, institutionalization, comorbidities and death.

\section{Types of studies}

This umbrella review will consider quantitative systematic reviews, meta-analyses and pooled analyses identifying relevant scientific evidence related to the reliability, validity and capacity to detect 
pre-frail and frail conditions, and the predictive accuracy of available screening tools for frailty in older adults.

\section{Search strategy}

The search strategy will aim to find published systematic reviews and meta-analyses. A three-step search strategy will be utilized in this umbrella review. An initial limited search of MEDLINE and CINAHL will be undertaken followed by an analysis of the text words contained in the title and abstract, and of the index terms used to describe the articles. A second search using all identified keywords and index terms will then be undertaken across all included databases. Thirdly, the reference list of all identified reports and articles will be searched for additional studies. Studies published in English from 2001 will be considered for inclusion in this review as 2001 was the year of publication of Fried's ${ }^{8}$ paper that was shown to be seminal for research on frailty condition.

Studies in other languages or outside the timeframe selected will be excluded.

The databases to be searched include:

MedicLatina, CINAHL Complete, MEDLINE via EBSCOhost Web, Scielo - Scientific Electronic Library Online, Cochrane Database of Systematic Reviews (CDSR), Centre for Reviews and Dissemination Databases (Database of Reviews of Effects [DARE]), PROSPERO register and Joanna Briggs Institute (JBI) Library of Systematic Reviews and Implementation Reports.

The search for unpublished studies will include:

Grey Literature Report (The New York Academy of Medicine), ProQuest - Nursing and Allied Health Source Dissertations.

Initial keywords to be used will be:

review, meta-analysis, pre-frailty, frailty, diagnostic test, assessment, accuracy, clinical risk stratification instruments, screening, sensitivity, specificity, reliability validity, positive predictive value and negative predictive value.

Papers will be assessed for relevance utilizing the title, abstract and index terms, including papers found from searching reference lists of relevant papers. Screening will be conducted independently by two reviewers.

\section{Assessment of methodological quality}

Papers selected for retrieval will be assessed by two independent reviewers for methodological validity prior to inclusion in the review using standardized critical appraisal instruments from the Joanna Briggs Institute System for the Unified Management, Assessment and Review Instrument (JBI-SUMARI) and the Joanna Briggs Institute Reviewers' Manual 2014 ${ }^{29}$ (Appendix I). Any disagreements that arise between the reviewers will be resolved through discussion, or with a third reviewer.

\section{Data extraction}

Data will be extracted from papers included in the review independently by two reviewers, using the standardized data extraction tool from the JBI instrument designed for umbrella reviews (Appendix II). Data extracted will include specific details about type of review (i.e. meta-analysis - sensitivity, specificity, true positives [TP], false positives [FP], true negatives [TN] and false negatives [FN] are 
taken directly from the source papers or narrative summary), countries where the studies were conducted, number of studies in the review, instrument references, participants (number, age group), outcomes identified (type/characteristics), length and completeness of follow-up, primary outcome measures and specific objectives. For missing information or to clarify unclear data, the authors of this umbrella review will endeavor to contact the author(s) of the paper in question to seek clarification. Any disagreements that arise between the reviewers will be resolved through discussion, or with a third reviewer.

\section{Data summary}

There may be an overlap of included studies in the identified systematic reviews, which will be apparent on full text examination. When this occurs, the frequency of overlap of studies will be noted.

Data extracted from selected reviews related to the identified quality of screening tools (specified in Types of outcomes section) will be tabulated and accompanied by a narrative to address the review questions in line with the inclusion criteria. All results will be subject to double data entry. Tabular presentation of quantitative results describing the reliability, validity, capacity to detect pre-frail and frail conditions, and the predictive accuracy of available screening tools will be presented with supporting interpretation. Tables will include a high level of detail for each included systematic review, including: instrument references, number of studies, total number of participants, heterogeneity of results, setting, age and co-morbidity. ${ }^{29}$

The results of the review will be provided in tabular format in a "Summary of Evidence" table that consists of instrument references and estimates included in the systematic review(s). The criteria that will be evaluated include methodological limitations, consistency, risk of bias and relevance to the study population. ${ }^{29}$

It is also important to note that the reviewers are planning to undertake conversion of data where possible to facilitate further analysis. Positive and negative likelihood ratios, odds ratios and 95\% confidence intervals will be recalculated. The data will be displayed on forest and summary receiver operating characteristic (ROC) plots. Heterogeneity will be assessed statistically using the standard Chi-square and also explored using subgroup analyses based on the different study designs included in this review. To perform additional analysis and plots, the Stata Statistical Software (version 11.1. College Station, TX: StataCorp LP) will be used.

\section{Conflicts of interest}

The authors have no conflicts of interest to declare.

\section{Acknowledgements}

This protocol is part of the project/joint action "664367/FOCUS" which has received funding from the European Union's Health Programme (2014-2020).

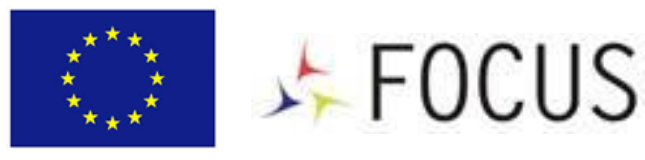

The authors acknowledge the contribution of other members of Focus project: Alessandro Nobili and Barbara D'Avanzo (IRCCS Istituto Di RicercheFarmacologiche "Mario Negri”), Ana González Segura 
and Enrique de la Cruz Martínez (EVERIS Spain S.L.U), Ana M. Martínez-Arroyo, Vicente Gil and Vicente Llorens (ESAM Tecnología S.L.), Donata Kurpas and Maria Bujnowska (Wroclaw Medical University), Federico Germini (Fondazione IRCCS Cà Granda - Ospedale Maggiore Policlinico), James Brown ${ }^{\dagger}$, Lex van Velsen (Roessingh Research and Development) and Rachel Shaw (Aston Research Centre for Healthy Ageing, Aston University) who were involved in decision-making and planning of the FOCUS project, and development of PICO questions and structuring of PICO components for this protocol.

The authors also thank Eduardo Santos for his contribution to the protocol development. 


\section{References}

1. Rockwood K, Fox RA, Stolee P, et al. Frailty in elderly people: An evolving concept. Can Med Assoc J 1994;150(4):489-495.

2. Fried LP, Ferrucci L, Darer J, et al. Untangling the concepts of disability, frailty, and comorbidity: implications for improved targeting and care. J Gerontol A Biol Sci Med Sci 2004;59(3):255-263.

3. Rockwood K. What would make a definition of frailty successful? Commentaries. Age Ageing 2005;34:432-434.

4. Sternberg SA, Schwartz AW, Karunananthan S, et al. The identification of frailty: A systematic literature review. Progress in Geriatrics 2011;59(11):2129-2139.

5. Le Maguet $\mathrm{P}$, Roquilly A, Lasocki S, et al. (2014). Prevalence and impact of frailty on mortality in elderly ICU patients: a prospective, multicenter, observational study. Intensive Care Med 2014;40(5):674-682.

6. Arya S, Kim SI, Duwayri Y, et al. Frailty increases the risk of 30-day mortality, morbidity, and failure to rescue after elective abdominal aortic aneurysm repair independent of age and comorbidities. J Vasc Surg 2015;61(2):324-331.

7. Lahousse L, Maes B, Ziere G, et al. Adverse outcomes of frailty in the elderly: the Rotterdam Study. Eur J Epidemiol 2014;29:419-427.

8. Fried LP, Tangen CM, Walston J, et al. Frailty in older adults: evidence for a phenotype. J Gerontol A Biol Med Sci 2001;56(3):M146-M156.

9. Langlois F, Vu TTM, Kergoat MJ, et al. The multiple dimensions of frailty: physical capacity, cognition, and quality of life. International Psychogeriatrics 2012;24(9):1429-1436.

10. Ávila-Funes, JA, Amieva $\mathrm{H}$, Barberger-Gateau $\mathrm{P}$, et al. Cognitive Impairment Improves the Predictive Validity of the Phenotype of Frailty for Adverse Health Outcomes: The Three-City Study. J Am Geriatr Soc 2009;57(3):453-461.

11. Collard, RM, Comijs HC, Naarding P, et al. Frailty as a Predictor of the Incidence and Course of Depressed Mood. J Am Med Direct Ass 2015;16(6):509-514.

12. Rodriguez-Manas L, Feart C, Mann G, et al. Searching for an operational definition of frailty: a Delphi method based consensus statement: the frailty operative definition-consensus conference project. J Gerontol A Biol Sci Med Sci 2013;68(1):62-67.

13. Rockwood K, Mitnitski A. Frailty defined by deficit accumulation and geriatric medicine defined by frailty. Clin Geriatr Med 2011;27(1):17-26.

14. Collard RM, Boter $\mathrm{H}$, Schoevers RA, et al. Prevalence of frailty in community-dwelling older persons: A systematic review. J Am Geriatr Soc 2012;60(8):1487-1492.

15. Lang PO, Michel JP, Zekry D. Frailty syndrome: a transitional state in a dynamic process. Gerontology 2009;55(5):539-549.

16. Ferrucci L, Windham BG, Fried LP. Frailty in older persons. Genus (2005):39-53.

17. Varadhan R, Seplaki CS, Xue QL, et al. Stimulus-response paradigm for characterizing the loss of resilience in homeostatic regulation associated with frailty. Mech Ageing Dev 2008;129(11):666-670. 
18. Clegg A, Young J, lliffe S, et al. Frailty in elderly people. Lancet 2013; 381(9868):752-762.

19. Bergman H, Ferrucci L, Guralnik J, et al. Frailty: an emerging research and clinical paradigm issues and controversies. J. Gerontol. A: Biol. Sci. Med. Sci. 2007;62(7):731-737.

20. Topinková E. Aging, Disability and Frailty. Ann Nutr Metab 2008;52(suppl 1):6-11.

21. Gobbens R, van Assen M, Luijkx K, et al. Determinants of frailty. J Am Med Dir Assoc 2010;11(5):356-364.

22. Cameron ID, Fairhall N, Langron $\mathrm{C}$, et al. A multifactorial interdisciplinary intervention reduces frailty in older people: randomized trial. BMC Med 2013;11:65.

23. Cesari M, Vellas B, Hsu F-C, et al. A Physical Activity Intervention to Treat the Frailty Syndrome in Older Persons - Results From the LIFE-P Study. J Gerontol A Biol Sci Med Sci 2015;70(2):216-222.

24. Pulignano G, Del Sindaco D, Di Lenarda A, et al. Usefulness of frailty profile for targeting older heart failure patients in disease management programs: a cost-effectiveness, pilot study. $\mathrm{J}$ Cardiovasc Med 2010;11(10):739-747.

25. Eklund K, Wilhelmson K, Gustafsson $\mathrm{H}$, et al. One-year outcome of frailty indicators and activities of daily living following the randomized controlled trial; "Continuum of care for frail older people". BMC Geriatr 2013;13:76.

26. Rockwood K, Andrew M, Mitnitski A. A comparison of two approaches to measuring frailty in elderly people. J Gerontol A Biol Sci Med Sci 2007;62(7):738-743.

27. Basic D, Shanley Ch. Frailty in older inpatients population: Using the clinical frailty scale to predict patient outcomes. J Aging Health 2015;27(4):670-685.

28. Pijpers E, Ferreira I, Stehouwer C, et al. The frailty dilemma. Review of the predictive accuracy of major frailty scores. Eur J Intern Med 2012;23(2):118-123.

29. The Joanna Briggs Institute. Joanna Briggs Institute Reviewers' Manual: Methodology for JBI Umbrella Reviews. Adelaide: The Joanna Briggs Institute; 2014.

30. Rubenstein LZ, Stuck AE, Siu AL, et al. Impacts of geriatric evaluation and management programs on defined outcomes: overview of the evidence. J Am Geriatr Soc 1991;39(9 Pt 2):8S16S; discussion 17S-18S. 


\section{Appendix I: Appraisal instruments}

\section{JBI critical appraisal checklist for systematic reviews and research syntheses}

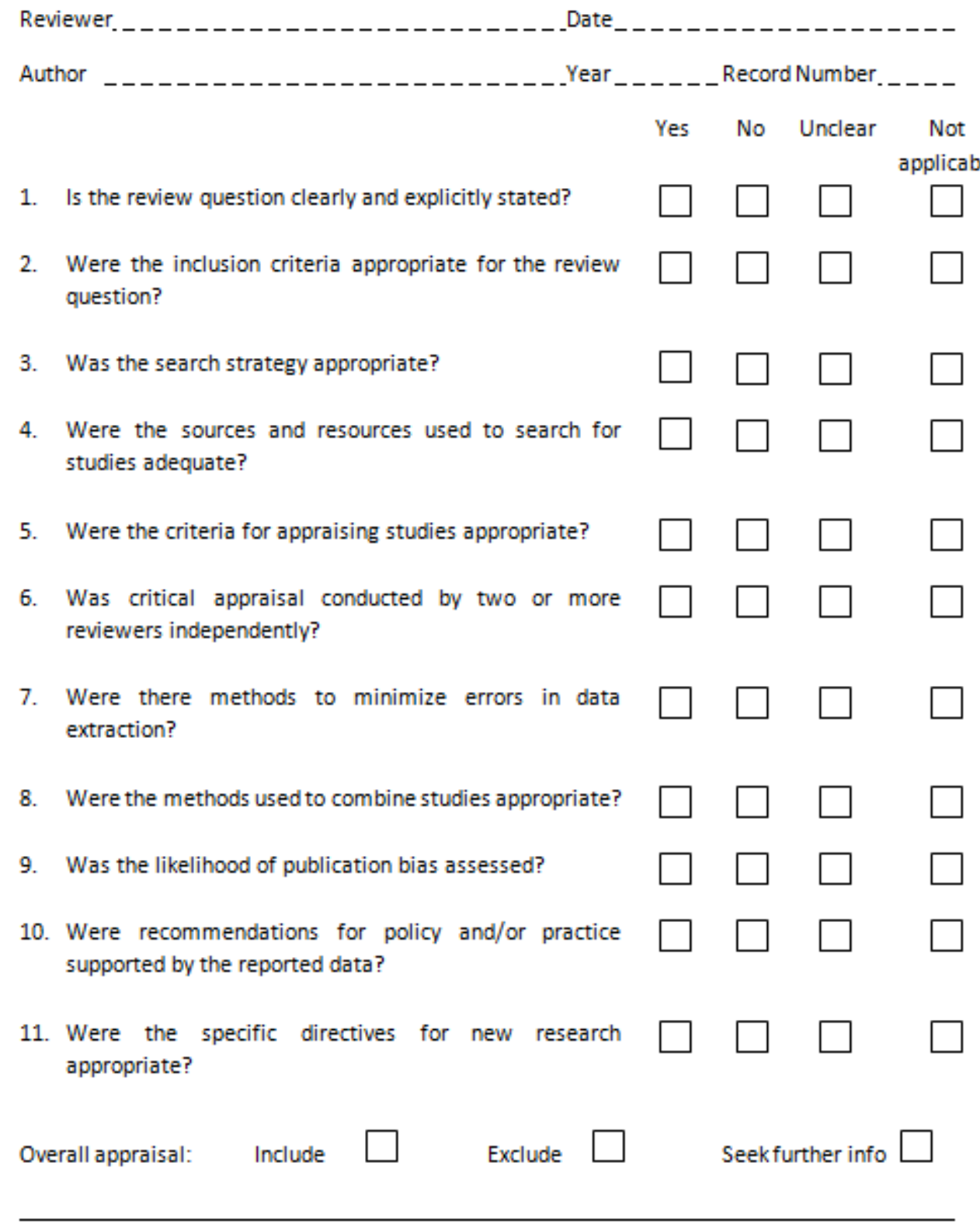




\section{Appendix II: Data extraction instruments}

JBI data extraction form for review for systematic reviews and research syntheses

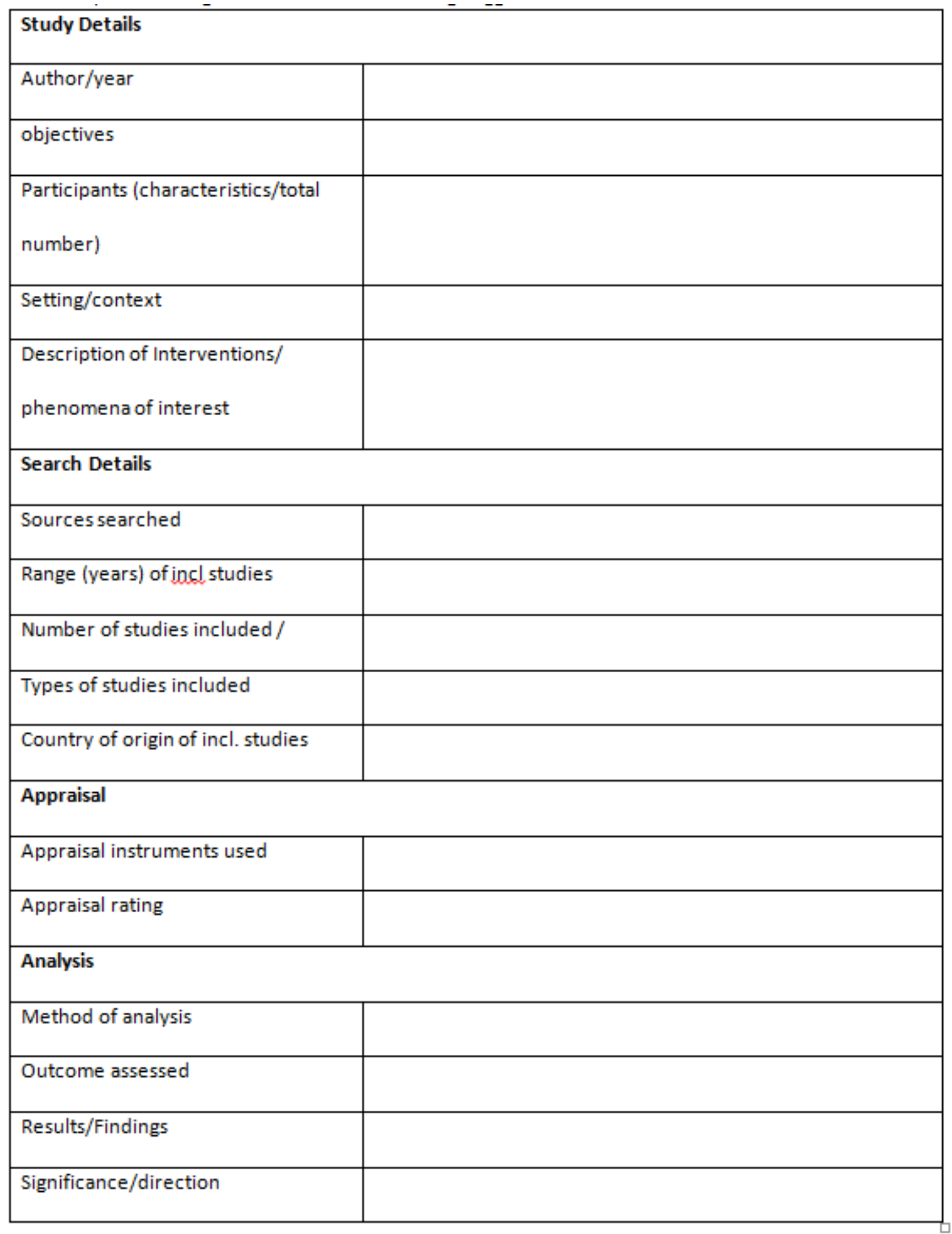

